\title{
Ultrasonographic and Clinical Assessment of Peripheral Enthesitis in Patients with Psoriatic Arthritis, Psoriasis, and Fibromyalgia Syndrome: The ULISSE Study
}

\author{
Pierluigi Macchioni, Carlo Salvarani, Niccolò Possemato, Marwin Gutierrez, Walter Grassi, \\ Stefania Gasparini, Carlo Perricone, Fabio Massimo Perrotta ${ }^{\circledR}$, Rosa Daniela Grembiale, \\ Caterina Bruno, Cesare Tripolino, Marcello Govoni, Giovanni Ciancio, Ilaria Farina, \\ Roberta Ramonda, Paola Frallonardo, Francesca Desiati, Raffaele Scarpa, Luisa Costa, \\ Alen Zabotti (i), Salvatore De Vita, Rita Maria D’Attino, Giuliana Gualberti (D), Rocco Merolla, \\ Umberto di Luzio Paparatti, Raffaella Aldigeri (D, and Antonio Marchesoni
}

\begin{abstract}
Objective. The purpose of the ULISSE study was to evaluate the prevalence of clinical and ultrasonographic (US) entheseal involvement in patients with psoriatic arthritis (PsA), psoriasis, and fibromyalgia syndrome (FMS).

Methods. In this cross-sectional multicenter study, patients with PsA and psoriasis (not taking systemic therapy) and FMS underwent a clinical evaluation of the entheses, and a B-mode and power Doppler examination of 6 pairs of entheses.

Results. The study analyzed 140 patients with PsA, 51 with psoriasis, and 51 with FMS. Clinical and US examinations were performed in 1960 and 1680 entheses in the PsA group, and 714 and 612 entheses both in the psoriasis group and in the FMS group. In both per-patient and per-enthesis evaluation, the frequency of entheseal tenderness was higher in patients with FMS ( $92 \%$ of the patients and $46 \%$ of the entheses, compared with $66 \% / 23 \%$ in the PsA group and 59\%/18\% in the psoriasis group). With US examination, signs of entheseal involvement were more frequent in both the per-patient and per-enthesis evaluation in PsA and psoriasis (about $90 \%$ of patients in both the PsA and psoriasis groups and $75 \%$ of patients in the FMS group had at least 1 site affected, and 54\%, $41 \%$, and $27 \%$ of the pairs of entheses in, respectively, PsA, psoriasis, and FMS patients showed at least 1 enthesis involved).

Conclusion. The ULISSE study indicated that enthesitis is a common feature in patients with PsA, those with psoriasis, and in those with FMS if only clinical examination is used. US entheseal assessment showed findings more consistent with the 3 disorders. (First Release March 15 2019; J Rheumatol 2019;46:904-11; doi:10.3899/jrheum.171411)
\end{abstract}

Key Indexing Terms:

PSORIATIC ARTHRITIS PSORIASIS

FIBROMYALGIA SYNDROME ENTHESITIS ULTRASONOGRAPHY

\begin{abstract}
From the Azienda USL-Institute for Research and Health Care (IRCCS) di Reggio Emilia; Università di Modena e Reggio Emilia, Reggio Emilia, Italy; Division of MSK and Rheumatic Disorders, National Institute of Rehabilitation, Mexico City, Mexico; Rheumatology Unit, Università Politecnica delle Marche Ospedale C. Urbani, Jesi; Rheumatology Unit, Department of Internal Medicine and Medical Specialties, Sapienza University, Rome; Dipartimento di Medicina e Scienze della salute, Università degli studi del Molise, Campobasso; Rheumatology Research Unit, Dipartimento di Scienze della Salute, Università "Magna Graecia" di Catanzaro, Catanzaro; Rheumatology Unit, Department of Medical Sciences, University of Ferrara; Azienda Ospedaliero-Universitaria S. Anna - Ferrara; Rheumatology Unit, Department of Medicine DIMED, University of Padua, Padua; Day Hospital of Rheumatology, Azienda Socio Sanitaria Territoriale (ASST) Gaetano Pini-Centro Specialistico Ortopedico (CTO), Milan; Rheumatology Unit, Department of Clinical Medicine and Surgery, University Federico II, Naples; Rheumatology Clinic, Department of Medical and Biological Sciences, University Hospital "Santa Maria della Misericordia," Udine; AbbVie Srl, Rome;
\end{abstract}

Department of Medicine and Surgery, University of Parma, Parma, Italy. R.M.D'Attino, G. Gualberti, and R. Merolla are employees of AbbVie and may own AbbVie stocks/options. U. di Luzio Paparatti was an employee of AbbVie and may own AbbVie stocks/options. AbbVie participated in the design, study conduct, and financial support for the study, as well as in interpretation of the data, review, and approval of the manuscript. Dr. C. Salvarani has acted as a consultant for AbbVie, MSD, Pfizer Inc, Roche, Celgene, and Novartis. Dr. W. Grassi has received honoraria and speaker fees from AbbVie, Roche, BMS, Pfizer, UCB, and MSD. Dr. M. Gutierrez has attended advisory board meetings, held scientific consultancies, and has obtained consulting fees from AbbVie, UCB Pharma, Esaote SpA, Bristol-Myers Squibb, Novartis, and Merck Sharp \& Dohme. P. Macchioni, MD, Azienda USL-IRCCS di Reggio Emilia; C. Salvarani, $M D$, Azienda USL-IRCCS di Reggio Emilia, and Università di Modena e Reggio Emilia; N. Possemato, MD, Azienda USL-IRCCS di Reggio Emilia; M. Gutierrez, MD, Division of MSK and Rheumatic Disorders, National Institute of Rehabilitation; W. Grassi, MD, Rheumatology Unit, Università Politecnica delle Marche Ospedale C. Urbani; S. Gasparini, 
Psoriatic arthritis (PsA) is a chronic inflammatory musculoskeletal disorder affecting patients with psoriasis or with a familial predisposition to psoriasis, which belongs to the spondyloarthritis (SpA) family of inflammatory rheumatic diseases ${ }^{1}$. Patients with PsA present with various combinations of peripheral joint synovitis, dactylitis, enthesitis, and spondylitis. Enthesitis is considered the hallmark of the SpA and occurs frequently in patients with PsA. It may involve few or many sites, superficial or deep entheses, and it may be asymptomatic or very painful and disabling $2,3,4,5,6,7,8$.

Clinical assessment of enthesitis is often difficult because of the frequent lack of apparent signs of inflammation and the involvement of clinically inaccessible sites. A number of studies have described the use of ultrasonography (US) as a useful tool to evaluate enthesitis in patients with SpA and $\mathrm{PsA}^{9-18}$. These studies observed a low concordance rate between clinical and US examination and a high prevalence of entheseal abnormalities in patients with psoriasis without arthritis.

Fibromyalgia syndrome (FMS) is characterized by widespread chronic pain, fatigue, sleep disturbances, and various combinations of somatic manifestations. On clinical examination, patients with FMS usually present with musculoskeletal tenderness everywhere, including the entheseal

MD, Rheumatology Unit, Università Politecnica delle Marche Ospedale C. Urbani; C. Perricone, MD, Rheumatology Unit, Department of Internal Medicine and Medical Specialties, Sapienza University; F.M. Perrotta, $M D$, Dipartimento di Medicina e Scienze della Salute, Università degli studi del Molise; R.D. Grembiale, MD, Rheumatology Research Unit, Dipartimento di Scienze della Salute, Università "Magna Graecia” di Catanzaro; C. Bruno, MD, Rheumatology Research Unit, Dipartimento di Scienze della Salute, Università "Magna Graecia” di Catanzaro; C. Tripolino, MD, Rheumatology Research Unit, Dipartimento di Scienze della Salute, Università “Magna Graecia” di Catanzaro; M. Govoni, MD, Rheumatology Unit, Department of Medical Sciences, University of Ferrara, and Azienda Ospedaliero-Universitaria S. Anna; G. Ciancio, MD, Rheumatology Unit, Department of Medical Sciences, University of Ferrara, and Azienda Ospedaliero-Universitaria S. Anna; I. Farina, MD, Rheumatology Unit, Department of Medical Sciences, University of Ferrara, and Azienda Ospedaliero-Universitaria S. Anna; R. Ramonda, $M D$, Rheumatology Unit, Department of Medicine DIMED, University of Padua; P. Frallonardo, MD, Rheumatology Unit, Department of Medicine DIMED, University of Padua; F. Desiati, MD, Day Hospital of Rheumatology, ASST Gaetano Pini-CTO; R. Scarpa, MD, Rheumatology Unit, Department of Clinical Medicine and Surgery, University Federico II; L. Costa, MD, Rheumatology Unit, Department of Clinical Medicine and Surgery, University Federico II; A. Zabotti, MD, Rheumatology Clinic, Department of Medical and Biological Sciences, University Hospital "Santa Maria della Misericordia"; S. De Vita, MD, Rheumatology Clinic, Department of Medical and Biological Sciences, University Hospital "Santa Maria della Misericordia”; R.M. D'Attino, MD, AbbVie Srl; G. Gualberti, PhD, AbbVie Srl; R. Merolla, MD, AbbVie Srl; U. di Luzio Paparatti, MD, AbbVie Srl; R. Aldigeri, BSc, Department of Medicine and Surgery, University of Parma; A. Marchesoni, MD, Day Hospital of Rheumatology, ASST Gaetano Pini-CTO.

Address correspondence to Dr. A. Marchesoni, Day Hospital of Rheumatology, ASST Gaetano Pini-CTO, Piazza Andrea Ferrari 1, 20122

Milan, Italy.E-mail: Antonio.Marchesoni@asst-pini-cto.it

Full Release Article. For details see Reprints and Permissions at jrheum.org

Accepted for publication October 29, 2018 sites. One study showed that the number of tender entheses was significantly higher in FMS than in patients with PsA ${ }^{19}$ : patients with FMS may be difficult to distinguish from patients with SpA with only polyenthesitis.

The primary objective of our study was to evaluate the prevalence of clinical and US signs of entheseal involvement in patients with PsA compared with patients with psoriasis and FMS. Secondary objectives were to determine the sensitivity and specificity of B-mode and power Doppler (PD) US signs of enthesitis versus clinical signs, and to evaluate the correlation of disease activity with the US signs of enthesitis in patients with PsA.

\section{MATERIALS AND METHODS}

Study design. This cross-sectional multicenter study was conducted in 10 Italian rheumatologic centers collaborating with dermatologic centers with a recognized expertise in PsA and US imaging. The study was approved by the local ethics committees of all of the participating centers according to Italian current legislation on epidemiological studies (First approval: prot $\mathrm{n}$. 11/2011 dated 15 Dec 2011 of the EC Comitato Etico della provincia di Ferrara).

Study populations. Adult patients (age 18-65 yrs) were enrolled if they had PsA according to the ClASsification for Psoriatic ARthritis (CASPAR) criteria $^{20}$, psoriasis diagnosed by a dermatologist, or a diagnosis of FMS based on a rheumatologist's opinion. Written consent was required, according to the Declaration of Helsinki.

Patients with PsA were excluded from the study if they had concomitant FMS, psoriasis and FMS, or any musculoskeletal complaint; patients with FMS were excluded if they had any form of arthritis. Other exclusion criteria were presence or history of other concomitant rheumatic inflammatory disorders and inflammatory bowel diseases, presence of tendinitis due to overuse or physical stress, and recent articular injury. Patients receiving current treatment with systemic corticosteroids, disease-modifying antirheumatic drugs (DMARD), including biologics, and intraarticular or intraentheseal corticosteroids during the past 4 weeks were excluded, as were patients participating in clinical trials or with any medical condition that could have jeopardized their ability to participate in the study.

Collection of clinical data. Personal and clinical data were collected by rheumatologists using an electronic case report form. Data were collected including patient demographics, anthropometry, lifestyle, social profile, date of disease onset and diagnosis, concomitant diseases, and medications. Clinical examination consisted of joint count (28 joints for swelling and tenderness) and enthesis evaluation. The following entheses were examined for tenderness and swelling bilaterally: common extensor tendon (CET) insertion on the lateral epicondyle of the humerus, quadriceps tendon (QT), patellar tendon (PT), tibial tuberosity, knee medial collateral ligament (MCL), Achilles tendon (AT), and plantar fascia (PF) insertion on the calcaneus. These scores were calculated: Leeds Enthesitis Index (LEI) ${ }^{21}$, Maastricht Ankylosing Spondylitis Enthesitis Score (MASES)22, and the 28 -joint count Disease Activity Score (DAS28) ${ }^{23}$. In addition to clinical data, C-reactive protein and erythrocyte sedimentation rate (ESR) levels were measured.

Collection of US data. US was performed in B-mode and PD mode. All centers used the same US machine (ESAOTE MyLab70) equipped with 18-6 $\mathrm{MHz}$ and 13-5 MHz multifrequency linear probe. To standardize the US evaluation of the entheseal sites, all the sonographers attended a training meeting. In addition, they were given a booklet with standard US imaging instructions. All the operators were experienced in musculoskeletal US and blinded to diagnosis and clinical findings. All the scan images were recorded for digital imaging. The US evaluation was performed within 2 weeks of the clinical evaluation. The following entheseal sites were examined bilaterally

Personal non-commercial use only. The Journal of Rheumatology Copyright @ 2019 . All rights reserved. 
in transverse and longitudinally according to a standard protocol: CET, QT, PT, MCL, AT, and PF. In B-mode assessment, all the following abnormal findings were recorded: entheseal thickening measured at $2 \mathrm{~mm}$ proximal to the bony contour (abnormality definitions: quadriceps tendon $>6.1 \mathrm{~mm}$, proximal and distal patellar ligament $>4 \mathrm{~mm}$, Achilles tendon $>5.29 \mathrm{~mm}$, plantar aponeurosis $>4.4 \mathrm{~mm})^{10}$, entheseal hypoechogenicity (defined as loss of normal fibrillar architecture), peritendon hypoechogenicity (defined as presence of blurring contours of tendon), bony erosions (defined as a cortical break with a stepdown contour defect, seen in 2 perpendicular planes, at the insertion of the enthesis to the bone), enthesophytes (defined as bony prominence at the end of the normal bone contour, seen in 2 perpendicular planes, with or without acoustic shadow), and enlargement of bursae (defined as the presence of enlarged bursae at their anatomic sites as a well-circumscribed localized anechoic or hypoechoic area at the site of an anatomic bursa compressible by the transducer). These lesions were scored as 1 or 0 if present or absent. Entheseal thickening, entheseal hypoechogenicity, peritendon hypoechogenicity, and bursal enlargement were considered acute lesions. Bony erosions, calcifications, and enthesophytes were considered chronic lesions. Entheses were scored globally as 1 (presence of $\geq 1$ lesion) and separately as 1 for acute involvement (presence of $\geq 1$ acute lesion), and 1 for chronic involvement (presence of $\geq 1$ chronic lesion). Vascularization was examined using PD mode, standardized with a pulse repetition frequency of $750 \mathrm{~Hz}$ and a PD gain of $50-53 \mathrm{~dB}$. Vascularization was studied at the following areas: cortical bone insertion, body of tendon, bursa, and junction between the tendon and enthesis. The detection of vascularization in any of these areas was considered abnormal. Enthesis US vascularity was classified into 4 distinctive patterns according to the number of vessels involved: $0=$ none; $1=1$ to 3 vessels; $2=4$ to 5 vessels; $3=$ more than 5 vessels. The presence of PD signal $>1$ was considered indicative of an acute lesion.

Statistical analysis. Because this was a study intended to generate descriptive statistics only, no formal sample size calculation was performed; therefore, a total sample of 250 subjects was considered appropriate to provide preliminary indications. Descriptive statistics and differences among the 3 study groups were presented for continuous variables, and absolute and relative frequencies for categorical variables. Given the nature of the study, statistical comparisons were not needed. However, variables were compared to provide a better measure of the differences. For continuous variables, comparison among groups was tested by means of a t test or an analysis of variance for normally distributed variables, and a Mann-Whitney U test or Kruskal-Wallis test for the other variables. The chi-square test or the Fisher exact test were used for categorical variables as appropriate. Whenever necessary, normality was assessed by means of the Shapiro-Wilk test.

Sex, body mass index (BMI), age, disease duration, and diagnosis were correlated through linear regression models with US results, keeping male sex and FMS group as reference categories.

Frequencies and percentages of each distinct type of abnormality, without specifying the enthesis, were also analyzed. These percentages were calculated using the total number of possible abnormalities investigated per enthesis (10), multiplied by the 2 sides (right and left), multiplied by the total number of patients for each group. The sensitivity and specificity of US signs of enthesitis versus clinical examination were computed for each site. Positive predictive value, negative predictive value, and Cohen's $\kappa$ coefficient between US and clinical findings were also calculated, including confidence. An additional analysis of the results for the same sites was performed considering only PD data. Correlations with disease activity indices were performed only for patients with PsA. The level of significance was set at 0.05 .

Data were analyzed using the SAS system for Windows version 9.2 (SAS Institute) and SPSS v.22 (IBM Statistics).

\section{RESULTS}

The final study population included 242 patients: 140 with PsA, 51 with psoriasis, and 51 with FMS. Main demographic and general data of the 3 study populations are reported in
Table 1. Among the groups, statistically significant differences were found for sex (more female patients in the FMS group; $\mathrm{p}<0.0001$ ), disease duration (higher in the psoriasis group; $\mathrm{p}<0.0001$ ), BMI (lower in the FMS group; $\mathrm{p}=0.001$ ), and DAS28 (higher in the PsA group; $\mathrm{p}<0.0001$ ). Clinical evaluation. The global number of clinically evaluated entheses was 1960 in the PsA group, 714 for psoriasis, and 714 for FMS. Clinical signs (tenderness and/or swelling) of enthesitis were found in $92.2 \%$ of patients with FMS, $66.4 \%$ of those with PsA, and $58.8 \%$ of those with psoriasis. The percentages of entheses with clinical signs of enthesitis were $45.6 \%$ in FMS, 23.1\% in PsA, and $18.1 \%$ in psoriasis. AT was the only entheseal site with a similar percentage of involvement in patients with PsA and FMS (29.3\% and $25.5 \%$, respectively) and CET was the enthesis with the greatest difference between these 2 patient groups (38.6\% and $76.5 \%$, respectively). The median values of LEI and MASES are reported in Table 2.

US evaluation. The global number of US-evaluated entheses was 1680 in the PsA group, 612 in the psoriasis group, and 612 in the FMS group. Most patients had $\geq 1$ abnormality detected by US, including $92.1 \%$ with PsA, $90 \%$ with psoriasis, and $74.6 \%$ with FMS. Considering B-mode evaluation only, the results were similar, with $90 \%$ of patients with PsA, 92.2\% with psoriasis, and $62.7 \%$ with FMS having $\geq 1$ abnormality. Using PD-mode evaluation, $\geq 1$ abnormality was noted in $59.3 \%$ of patients with PsA, $47.1 \%$ with psoriasis, and $35.3 \%$ with FMS.

Grouping the entheses in pairs, $\mathrm{a} \geq 1$ US abnormality was observed in $53.7 \%$ of the pairs in patients with PsA, $41.2 \%$ in patients with psoriasis, and $27.4 \%$ in FMS. These percentages remained similar considering only the B-mode evaluation $(49.5 \%, 37.6 \%$, and $22.6 \%$, respectively), but decreased at the PD-mode evaluation $(19.2 \%, 12.7 \%$, and $7.8 \%$, respectively). The median B-mode and PD-mode scores are reported in Table 2. The number of abnormalities by PD assessment was similar in patients with PsA and psoriasis and higher in these 2 study populations than in the FMS population.

The models of linear regression created to evaluate which factors (among sex, diagnosis, age, BMI, and disease duration) were independently predictive of the highest US scores (Supplementary Tables 1-11, available with the online version of this article) yielded the following associations: PsA with total US score $\left(\mathrm{R}^{2}=0.130, \beta=6.383,95 \% \mathrm{CI}\right.$ $3.237-9.529, \mathrm{p}<0.001)$; PsA with PD score $\left(\mathrm{R}^{2}=0.059\right.$, $\beta=2.142,95 \%$ CI $0.554-3.731, p=0.008)$; PsA with B-mode score $\left(\mathrm{R}^{2}=0.155,95 \%\right.$ CI $\left.2.313-6.169, \mathrm{p}<0.001\right)$, age with B-mode score $(\beta=0.072,95 \%$ CI $0.003-0.141$, $\mathrm{p}=0.042)$, and BMI with B-mode score $(\beta=0.166,95 \%$ CI $0.018-0.314, \mathrm{p}=0.028)$; PsA with acute $\mathrm{B}$-mode score $\left(\mathrm{R}^{2}\right.$ $=0.114, \beta=2.504,95 \% \mathrm{CI} 0.974-4.035, \mathrm{p}=0.001)$ and $\mathrm{BMI}$ with acute B-mode score $(\beta=0.152,95 \%$ CI $0.035-0.270$, $\mathrm{p}=0.011)$; PsA with chronic B-mode score $\left(\mathrm{R}^{2}=0.156\right.$, $\beta=1.736,95 \%$ CI $0.841-2.631, p<0.001)$ and age with

Personal non-commercial use only. The Journal of Rheumatology Copyright (C) 2019. All rights reserved 
Table 1. Patient demographics.

\begin{tabular}{lcccc}
\hline Characteristics & PsA, $\mathrm{n}=140$ & Psoriasis, $\mathrm{n}=51$ & FMS, $\mathrm{n}=51$ & $\mathrm{p}$ \\
\hline Sex, M/F & $70 / 70$ & $23 / 28$ & $4 / 47$ & $<0.0001$ \\
Age, yrs & $48(40-58)$ & $51(44-57)$ & $50(42-57)$ & $\mathrm{ns}$ \\
Disease duration, yrs* & $5.6(1-8)$ & $12(6.0-23)$ & $3.5(2-10)$ & $<0.0001$ \\
BMI, kg/m & $25.6(22.0-29.1)$ & $27.1(24.3-30.4)$ & $23.4(21.5-24.7)$ & 0.001 \\
Comorbidities, $\mathrm{n}(\%)^{\dagger}$ & & & & \\
$\quad$ Cardiovascular & $30(21.43)$ & $15(29.41)$ & $11(21.57)$ & $\mathrm{ns}$ \\
$\quad$ Metabolic, nutrition & $18(12.86)$ & $7(13.75)$ & $5(9.8)$ & $\mathrm{ns}$ \\
$\quad$ Endocrine & $14(10)$ & $4(7.84)$ & $8(15.69)$ & $\mathrm{ns}$ \\
DAS28 & $3.05(2.29-4.06)$ & $2.30(1.67-2.84)$ & $2.49(2.07-4.02)$ & $<0.0001$ \\
ESR, mm/h & $12.0(6.0-22.0)$ & $10.0(6.0-14.0)$ & $7.0(5.0-14.2)$ & $\mathrm{ns}$ \\
CRP, mg/dl & $0.30(0.10-0.90)$ & $0.30(0.15-0.59)$ & $0.20(0.10-0.30)$ & $\mathrm{ns}$ \\
Smoking, $(\%)$ & $32(23)$ & $14(27)$ & $17(33)$ & $\mathrm{ns}$ \\
Alcohol, $\mathrm{n}(\%)$ & $63(45)$ & $15(29)$ & $19(37)$ & $\mathrm{ns}$ \\
Use of pain medications, & & & $6(11.76)$ & $<0.05$ \\
$\quad \mathrm{n}(\%)$ & $22(15.71)$ & 0 & & \\
\hline
\end{tabular}

Continuous variables are reported as median and interquartile range. $*$ Since symptoms onset. ${ }^{\dagger}$ Only comorbidities present in $>3 \%$ of patients are reported. BMI: body mass index; CRP: C-reactive protein; DAS28: 28-joint count Disease Activity Score; ESR: erythrocyte sedimentation rate; FMS: fibromyalgia syndrome; ns: not significant; PsA: psoriatic arthritis

Table 2. Scores of enthesitis (clinical and US) among different diagnoses.

\begin{tabular}{lcccc}
\hline Score Types & PsA, $\mathrm{n}=140$ & Psoriasis, $\mathrm{n}=51$ & FMS, $\mathrm{n}=51$ & $\mathrm{p}$ \\
\hline LEI painful & $1(0-2)$ & $0(0-2)$ & $3(2-4)$ & $<0.0001$ \\
LEI swollen & $0(0-0)$ & $0(0-0)$ & $0(0-0)$ & 0.03 \\
LEI painful + swollen & $1(0-3)$ & $0(0-2)$ & $3(2-4)$ & $<0.0001$ \\
MASES & $1(0-6)$ & $0(0-4)$ & $7(3-11)$ & $<0.0001$ \\
MASES upper body & $0(0-3)$ & $0(0-2)$ & $5(2-9)$ & $<0.0001$ \\
MASES legs & $0(0-2)$ & $0(0-1)$ & $1(0-3)$ & $\mathrm{ns}$ \\
B-mode chronic lesions* & $2(0-5)$ & $2(1-4)$ & $0(0-1)$ & $<0.0001$ \\
B-mode acute lesions** & $3(1-6.75)$ & $2(0-5)$ & $1(0-3)$ & $<0.0001$ \\
B-mode chronic + acute & & & & 0.0001 \\
$\quad$ lesions & $6(3.25-11)$ & $4(2-9)$ & $2(0-5)$ & 0.01 \\
PD abnormalities & $1(0-5)$ & $0(0-2)$ & $0(0-1)$ & \\
PD lesions, $\mathrm{n}(\%)$ & $83(59)$ & $24(47)$ & $18(35)$ & \\
$\quad+(1-3$ vessels $)$ & $38(27)$ & $15(29)$ & $11(22)$ & $4(8)$ \\
$\quad++(4-5$ vessels $)$ & $25(18)$ & $6(12)$ & $3(6)$ & \\
$+++(>5$ vessels $)$ & $20(14)$ & $3(6)$ & & \\
\hline
\end{tabular}

Scores are reported as median (25th percentile-75th percentile), except where indicated. * Bony erosions, calcifications, and enthesophytes. ** Entheseal thickening, entheseal hypoechogenicity, peritendon hypoechogenicity, and bursal enlargement. FMS: fibromyalgia syndrome; LEI: Leeds Enthesitis Index; MASES: Maastricht Ankylosing Spondylitis Enthesitis Score; PD: power Doppler; PsA: psoriatic arthritis; US: ultrasonography; ns: not significant.

chronic B-mode score $(\beta=0.077,95 \%$ CI $0.045-0.109$, $\mathrm{p}<0.001)$; PsA with AT B-mode score $\left(\mathrm{R}^{2}=0.095, \beta=1.131\right.$, 95\% CI 0.466-1.795, $\mathrm{p}=0.001)$; PsA with PT B-mode score $\left(\mathrm{R}^{2}=0.123, \beta=0.923,95 \% \mathrm{CI} 0.424-0.1 .422, \mathrm{p}<0.001\right)$ and BMI with PT B-mode score $(\beta=0.043,95 \%$ CI $0.005-0.081$, $\mathrm{p}=0.027)$; and PsA with QT B-mode score $\left(\mathrm{R}^{2}=0.045\right.$, $\beta=0.864,95 \%$ CI $0.233-1.495, p=0.007$ ).

Frequency of the US lesions found at the examined entheseal sites with B-mode and PD-mode US are reported in Table 3 and Table 4, respectively.

The most common abnormalities were enthesophytes and entheseal hypoechogenicity, which were found in $20.1 \%$ and $17.4 \%$ of patients with PsA, in $18.5 \%$ and $10.9 \%$ with psoriasis, and in $8 \%$ and $6.7 \%$ with FMS, respectively. Entheseal thickening, entheseal hypoechogenicity, peritendon hypoechogenicity, bone erosions, and enthesophytes and bursal enlargement were all more frequent in the entheses of patients with PsA than in those with FMS. Most of the B-mode lesions were more frequent in patients with psoriasis than in patients with FMS, especially entheseal thickening, entheseal hypoechogenicity, peritendon hypoechogenicity, and enthesophytes. 
Regarding abnormalities for individual entheses, changes were found most commonly at the AT and QT: $13 \%$ and $10.9 \%$ in the PsA group, $9.1 \%$ and $9.3 \%$ in the psoriasis group, and $4 \%$ and $5.5 \%$ in the FMS group, respectively. At the PD assessment, the AT in the PsA group (7.1\%), the PT in the psoriasis group (4.4\%), and the CET insertions in the FMS group (3.2\%) were the most common localizations (Supplementary Table 12, available with the online version of this article).

Globally, the presence of intraentheseal PD signal was higher at the cortical/bone insertion in patients with PsA than in patients with psoriasis and FMS. At the preinsertion level, patients with PsA and psoriasis had higher vascular signal than patients with FMS.

The results of the comparison between clinical and US assessment per patient and per enthesis are shown in Table 5. Sensitivity of the US assessment was higher in the perpatient analysis (ranging from 0.74 for FMS to 0.93 for psoriasis) than in the per-enthesis analysis (from 0.25 for FMS to 0.60 for PsA), while specificity was higher in the perenthesis analysis (from 0.48 for PsA to 0.71 for FMS) than in the per-patient analysis (from 0.06 for PsA to 0.25 for FMS). Globally, Cohen's $\kappa$ values were very low, ranging from -0.05 to 0.06 . As for the individual entheses, correlations were low at all sites. The highest Cohen's $\kappa$ value (0.243) was found in the AT with the PD evaluation in the per-patient analysis.

The comparison between PsA/psoriasis patients with or without entheseal clinical involvement indicated that patients with clinical enthesitis were more often female $(\mathrm{F} / \mathrm{M}=$ 70/42), had higher ESR values (mean ESR, 15 vs $11 \mathrm{~mm} / \mathrm{h}$ ), and higher DAS28 values (3.42 vs 2.83). Considering the US changes, the presence of lesions was more frequent in female patients $(\mathrm{F} / \mathrm{M}=70 / 35)$, and $\mathrm{BMI}$ was lower in patients with acute lesions (BMI $=23.1$ vs 25.9). In the PsA group, DAS28 values were higher in patients with MASES scores $>1$ (median, 3.42 vs 2.83); however, this was not the case for the entheseal B-mode or PD-mode scores $>1$.

\section{DISCUSSION}

This study provided a number of interesting findings about entheseal involvement in patients with PsA, psoriasis, and FMS. Using tenderness upon pressure on the entheseal site, enthesitis was more frequent in FMS than in patients with PsA and psoriasis. Given the abnormalities in pain perception typical of patients with FMS, this finding is not surprising and suggests that tenderness upon pressure is a poorly specific indicator of enthesitis. In distinguishing between PsA and FMS, these findings seem to indicate that patients with FMS have more tender entheses than do patients with PsA, especially in the upper body, confirming the results of a previous report ${ }^{19}$. The similar frequency of entheseal involvement recorded in patients with PsA and psoriasis was another interesting finding of this study, confirming that

Personal non-commercial use only. The Journal of Rheumatology Copyright @ 2019 . All rights reserved. 
Table 4. Frequency of the presence of PD-US changes at each examined entheseal site in the 3 populations.*

\begin{tabular}{|c|c|c|c|c|c|c|c|c|c|c|c|c|}
\hline \multirow[b]{2}{*}{ Entheseal site } & \multicolumn{3}{|c|}{ Cortical Bone Insertion } & \multicolumn{3}{|c|}{ Preinsertional Area } & \multicolumn{3}{|c|}{ Body of Tendon } & \multicolumn{3}{|c|}{ Bursa } \\
\hline & PsA & Psoriasis & FMS & PsA & Psoriasis & FMS & PsA & Psoriasis & FMS & PsA & Psoriasis & FMS \\
\hline \multicolumn{13}{|c|}{ Common extensor insertion } \\
\hline Quadriceps tendon & $10(7.1)$ & $5(9.8)$ & $1(2)$ & $8(5.7)$ & $3(5.9)$ & $2(3.9)$ & $7(5)$ & $2(3.9)$ & 0 & $5(3.6)$ & 0 & $1(2)$ \\
\hline Patellar tendon & $13(9.3)$ & $6(11.8)$ & $1(2)$ & $24(17.1)$ & $8(15.7)$ & $3(5.9)$ & $7(5)$ & $1(2)$ & $1(2)$ & $2(1.4)$ & $1(2)$ & 0 \\
\hline Medial collateral ligament & $18(12.9)$ & $2(3.9)$ & $1(2)$ & $8(5.7)$ & $1(2)$ & $1(2)$ & $12(8.6)$ & 0 & $1(2)$ & $1(0.7)$ & 0 & 0 \\
\hline on the calcaneus & 0 & 0 & 0 & $1(0.7)$ & $1(2)$ & 0 & $1(0.7)$ & 0 & 0 & 0 & 0 & 0 \\
\hline
\end{tabular}

Data are shown as no. patients (\%) and expressed in terms of affected patients. * PsA: $\mathrm{n}=140$ patients; psoriasis: $\mathrm{n}=51$ patients; FMS: $\mathrm{n}=51$ patients. FMS: fibromyalgia syndrome; PsA: psoriatic arthritis; PD-US: power Doppler ultrasonography.

Table 5. Comparison between clinical and US assessments in the 3 patient populations for both patients and entheses.

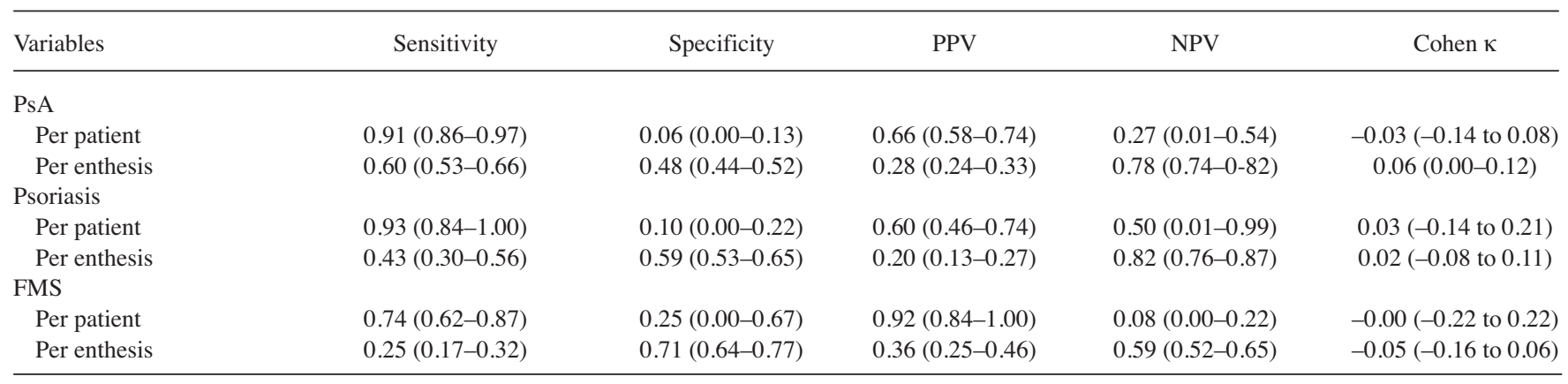

The findings are reported as mean values (lower-upper limit) of all of the entheses assessed both clinically and by US. Data were computed considering clinical assessment as the gold standard. FMS: fibromyalgia syndrome; NPV: negative predictive value; PPV: positive predictive value; PsA: psoriatic arthritis; US: ultrasonography.

patients with psoriasis may have enthesitis without having symptoms of PsA ${ }^{12}$. However, because about $16 \%$ of patients with PsA were taking pain medications, clinical signs of enthesitis in this group of patients might have been slightly underestimated. Swelling at the entheseal site, which was observed only in patients with PsA, was present in just 9\% of these patients.

Assessment by US showed a higher prevalence of signs of entheseal involvement in patients with PsA and psoriasis than in patients with FMS. Most of these US changes were seen by B-mode evaluation. PD-mode findings were much less frequent in all study populations, but they were still more frequent in patients with PsA, followed by psoriasis and FMS. The differences in sex, age, BMI, and disease duration among the 3 study populations might have biased the US findings. However, the linear regression models showed that PsA was the only factor independently associated with all of the US global scores. This disease was also associated with the B-mode lesions in the AT, PT, and QT. In contrast, no association was found for psoriasis and FMS. BMI and age showed a significant influence on the global B-mode score, indicating that these 2 factors have a role in the occurrence of entheseal lesions. At the level of the weight-bearing entheses, an association with the BMI was only found in the PT. These data indicate that, in our study populations, enthe- sitis evaluated by US was actually a feature of PsA, and in comparison, not of psoriasis and FMS. The stronger association between PsA and enthesitis was also indicated because patients with PsA had a higher number of most of the US changes, the only exception being osteophytes, which were similarly present in patients with psoriasis. A comparison with a control population of healthy subjects, however, would have likely found a higher amount of enthesitis in patients with psoriasis. Several studies have actually showed that US enthesitis is significantly more frequent in patients with psoriasis than in healthy controls ${ }^{13,17,18,24}$. In our study, patients with PsA or psoriasis showed a comparable number of entheseal sites with at least 1 US change, and numerically, the only relevant difference was in the pairs of entheses with a positive PD signal - there were more in PsA (about 19\%) than in psoriasis (about 13\%). The high number of US lesions found in patients with psoriasis was partly related to the BMI, which was higher in these patients, but that does not exclude the concept of asymptomatic enthesitis in these patients. Altogether, these findings confirm the relevance of enthesitis in PsA, which has been known for about 20 years ${ }^{25}$, and seem to support the hypothesis that the development of a higher degree of entheseal inflammation might be the pathogenetic mechanism underlying the switch from psoriasis to $\mathrm{PsA}^{26}$.

Another interesting finding of the US evaluation was that Personal non-commercial use only. The Journal of Rheumatology Copyright $\odot$ 2019. All rights reserved. 
signs of enthesitis were also found in patients with FMS, a disorder usually considered noninflammatory in nature. Because there was no control group of healthy subjects, the relevance of this result is unknown. US signs of enthesopathy, however, do not seem to be uncommon in healthy subjects, either ${ }^{27}$. Thus, in terms of diagnostic distinction between PsA and FMS, the presence of an individual US-positive enthesis is not helpful. In contrast, a high number of US-involved entheses makes PsA a more likely diagnosis; this finding is concordant with a previous study ${ }^{28}$. In addition, because the number of virtually all US changes was significantly higher in PsA than in FMS patients, an elevated entheseal US score points toward a diagnosis of PsA, as well as the presence of PD signal. This is especially true if the PD signal is grade $>1$ and localized at the cortical/bone insertion. The difference in US lesions between PsA and FMS was more evident in AT, and to a lesser extent, in QT and PT. AT and QT showed the highest number of US abnormalities. This finding seems to support the theory that biomechanical stress may have a role in the pathogenesis of PsA enthesitis ${ }^{29}$.

A secondary objective of our study was to compare US and clinical assessments. The results indicated that the concordance between the 2 assessments was very low, not only for chronic lesions, which may be asymptomatic, but also for B-mode acute abnormalities and PD-mode changes. This result confirms the already-reported poor correlation between clinical and US assessment of enthesitis in patients with PsA and $\mathrm{SpA}^{17,30,31,32}$.

Another secondary objective of our study was to examine the correlation between disease activity and entheseal involvement in patients with PsA. An association was found between clinical enthesitis and DAS28, which may be explained by the pain element shared by these 2 evaluations. In contrast, no correlation was seen between US enthesitis and DAS28, probably owing to low concordance between clinical and US findings of entheseal involvement. Another interesting result of our study was that females had more clinical and US entheseal involvement than men, a finding that might indicate a possible role of sex in the development of PsA phenotypes.

Our study had some limitations. First, the 3 groups were not matched for BMI, disease duration, and sex. Second, because patients receiving DMARD were excluded, the PsA and psoriasis populations consisted of patients with relatively mild disease. Third, no control group of healthy subjects was evaluated. Finally, because data on skin and nail involvement were not collected, a possible influence of the cutaneous condition on enthesitis could not be examined.

The ULISSE study showed that enthesitis is a common feature in patients with PsA and psoriasis. The main difference between these 2 conditions was that in patients with psoriasis, the US findings were less pronounced than in those with PsA. Enthesitis defined by tenderness upon pressure was very common in patients with FMS, a clear indication of the poor specificity of this definition of enthesitis. Regarding differential diagnosis, various clinical and US findings may help distinguish PsA from FSM. Entheseal sites exposed to a greater biomechanical stress were more likely to have US signs of enthesopathy, a finding with interesting pathogenetic implications. US assessment of the entheses is more objective than clinical evaluation, but a combination of clinical and US findings might be the best approach for a more reliable definition of enthesitis.

\section{IN MEMORIAM}

The contributions of Prof. Antonio Spadaro and Prof. Ignazio Olivieri were essential in conceiving and performing our study. We wish their memory to live on.

\section{ACKNOWLEDGMENT}

The authors thank Valeria Saragaglia of AbbVie for her vital support in project management.

\section{ONLINE SUPPLEMENT}

Supplementary material accompanies the online version of this article.

\section{REFERENCES}

1. Baraliakos X, Braun J. Spondyloarthritides. Best Pract Res Clin Rheumatol 2011;25:825-42.

2. McGonagle D, Gibbon W, Emery P. Classification of inflammatory arthritis by enthesitis. Lancet 1998;352:1137-40.

3. McGonagle D, Lories RJ, Tan AL, Benjamin M. The concept of a "synovio-entheseal complex" and its implications for understanding joint inflammation and damage in psoriatic arthritis and beyond. Arthritis Rheum 2007;56:2482-91.

4. Eshed I, Bollow M, McGonagle DG, Tan AL, Althoff CE, Asbach P, et al. MRI of enthesitis of the appendicular skeleton in spondyloarthritis. Ann Rheum Dis 2007;66:1553-9.

5. Benjamin M, McGonagle D. The enthesis organ concept and its relevance to the spondyloarthropathies. Adv Exp Med Bio 2009;649:57-70.

6. McGonagle DG, Helliwell P, Veale D. Enthesitis in psoriatic disease. Dermatology 2012;225:100-9.

7. McGonagle D, Tan AL. The enthesis in psoriatic arthritis. Clin Exp Rheumatol 2015;33(5 Suppl 93):S36-9.

8. Schett G, Lories RJ, D'Agostino MA, Elewaut D, Kirkham B, Soriano ER, et al. Enthesitis: from pathophysiology to treatment. Nat Rev Rheumatol 2017;13:731-41.

9. Olivieri I, Barozzi L, Padula A. Enthesiopathy: clinical manifestations, imaging and treatment. Baillieres Clin Rheumatol 1998;12:665-81.

10. Balint PV, Kane D, Wilson H, McInnes IB, Sturrock RD. Ultrasonography of entheseal insertions in the lower limb in spondyloarthropathy. Ann Rheum Dis 2002;61:905-10.

11. D'Agostino M, Said-Nahal R, Hacquard-Bouder C, Brasseur JL, Dougados M, Breban M. Assessment of peripheral enthesitis in the spondylarthropathies by ultrasonography combined with power Doppler. A cross-sectional study. Arthritis Rheum 2003;48:523-33.

12. Husic R, Gretler J, Felber A, Graninger WB, Duftner C, Hermann J, et al. Disparity between ultrasound and clinical findings in psoriatic arthritis. Ann Rheum Dis 2014;73:1529-36.

13. Gisondi P, Tinazzi I, El-Dalati G, Gallo M, Biasi D, Barbara LM, et al. Lower limb enthesopathy in patients with psoriasis without clinical signs of arthropathy: a hospital-based case-control study. Ann Rheum Dis 2008;67:26-30.

Personal non-commercial use only. The Journal of Rheumatology Copyright (C) 2019. All rights reserved. 
14. Gandjbakhch F, Terslev L, Joshua F, Wakefield RJ, Naredo E, D'Agostino MA. Ultrasound in the evaluation of enthesitis: status and perspectives. Arthritis Res Ther 2011;13:R188.

15. de Miguel E, Cobo T, Munoz-Fernandez S, Naredo E, Uson J, Acebes JC, et al. Validity of enthesis ultrasound assessment in spondyloarthropathy. Ann Rheum Dis 2009;68:169-74.

16. de Miguel E, Munoz-Fernandez S, Castillo C, Cobo-Ibanez T, Martin-Mola E. Diagnostic accuracy of enthesis ultrasound in the diagnosis of early spondyloarthritis. Ann Rheum Dis 2011;70:434-9.

17. Naredo E, Moller I, de Miguel E, Batlle-Gualda E, Acebes C, Brito E, et al. High prevalence of ultrasonographic synovitis and enthesopathy in patients with psoriasis without psoriatic arthritis: a prospective case-control study. Rheumatology 2011;50:1838-48.

18. Gutierrez M, Filippucci E, De Angelis R, Salaffi F, Filosa G, Ruta S, et al. Subclinical entheseal involvement in patients with psoriasis: an ultrasound study. Semin Arthritis Rheum 2011;40:407-12.

19. Marchesoni A, Atzeni F, Spadaro A, Lubrano E, Provenzano G, Cauli A, et al. Identification of the clinical findings distinguishing psoriatic arthritis and fibromyalgia. J Rheumatol 2012;39:849-55

20. Taylor W, Gladman D, Helliwell P, Marchesoni A, Mease P, Mielants H. Classification criteria for psoriatic arthritis: development of new criteria from a large international study. Arthritis Rheum 2006;54:2665-73.

21. Healy PJ, Helliwell PS. Measuring clinical enthesitis in psoriatic arthritis: assessment of existing measures and development of an instrument specific to psoriatic arthritis. Arthritis Rheum 2008;59:686-91.

22. Heuft-Dorenbosch L, Spoorenberg A, van Tubergen A, Landewé R, van ver Tempel $\mathrm{H}$, Mielants $\mathrm{H}$, et al. Assessment of enthesitis in ankylosing spondylitis. Ann Rheum Dis 2003;62:127-32.

23. Prevoo ML, van 't Hof MA, Kuper HH, van Leeuwen MA, van de Putte LB, van Riel PL. Modified disease activity scores that include twenty-eight-joint counts. Development and validation in a prospective longitudinal study of patients with rheumatoid arthritis. Arthritis Rheum 1995;38:44-8.
24. Acquacalda E, Albert C, Montaudie H, Fontas E, Danre A, Roux $\mathrm{CH}$, et al. Ultrasound study of entheses in psoriasis patients with or without musculoskeletal symptoms: A prospective study. J Bone Spine 2015;82:267-71.

25. McGonagle D, Conaghan PG, Emery P. Psoriatic arthritis. A unified concept twenty years on. Arthritis Rheum 1999;42:1080-6.

26. Aydin SZ, Ash ZR, Tinazzi I, Castillo-Gallego C, Kwok C, Wilson $\mathrm{C}$, et al. The link between enthesitis and arthritis in psoriatic arthritis: a switch to a vascular phenotype at insertions may play a role in arthritis development. Ann Rheum Dis 2013;72:992-5.

27. D'Agostino MA, Iagnocco A, Aegerter $\mathrm{P}$, Kleyer A, Zwerina J, Perricone $\mathrm{C}$, et al. Does subclinical inflammation contribute to impairment of function of knee joints in aged individuals? High prevalence of ultrasound inflammatory findings. Rheumatology 2015;54:1622-9.

28. Marchesoni A, De Lucia O, Rotunno L, De Marco G, Manara M. Entheseal power-Doppler ultrasonography: a comparison of psoriatic arthritis and fibromyalgia. J Rheumatol Suppl. 2012 July;89:29-31.

29. Jacques P, Lambrecht S, Verheugen E, Pauwels E, Kollias G, Armaka M, et al. Proof of concept: enthesitis and new bone formation in spondyloarthritis are driven by mechanical strain and stromal cells. Ann Rheum Dis 2014;73:437-45.

30. Freeston JE, Coates LC, Helliwell PS, Hensor EM, Wakefield RJ, Emery $\mathrm{P}$, et al. Is there subclinical enthesitis in early psoriatic arthritis? A clinical comparison with power Doppler ultrasound. Arthritis Care Res 2012;64:1617-21.

31. Aydin SZ, Karadag O, Filippucci E, Atagunduz P, Akdogan A, Kalyoncu U, et al. Monitoring Achilles enthesitis in ankylosing spondylitis during TNF-alpha antagonist therapy: an ultrasound study. Rheumatology 2010;49:578-82.

32. Feydy A, Lavie-Brion MC, Gossec L, Lavie F, Guerini H, Nguyen $\mathrm{C}$, et al. Comparative study of MRI and power Doppler ultrasonography of the heel in patients with spondyloarthritis with and without heel pain and in controls. Ann Rheum Dis 2012; 71:498-503. 